\title{
Correction to: Consequences of policy incoherence: how Zambia's post-FCTC investment policy stimulated tobacco production
}

\author{
Ronald Labonté ${ }^{1} \cdot$ Raphael Lencucha ${ }^{2} \cdot$ Fastone Goma ${ }^{3} \cdot$ Richard Zulu $^{3}$. \\ Jeffrey Drope ${ }^{4,5}$
}

Published online: 10 July 2019

○) Springer Nature Limited 2019

\section{Correction to: Journal of Public Health Policy https://doi.org/10.1057/s41271-019-00171-8}

The paper "Consequences of policy incoherence: how Zambia's post-FCTC investment policy stimulated tobacco production" was published without including the acknowledgements:

This research was supported by the Office of the Director, National Institutes of Health (OD) and the National Cancer Institute (NCI) under Award Number R01TW010898; and the National Institute on Drug Abuse, the Fogarty International Center and NCI under Award Number R01DA035158.

Publisher's Note Springer Nature remains neutral with regard to jurisdictional claims in published maps and institutional affiliations.

The original article can be found online at https://doi.org/10.1057/s41271-019-00171-8.

Ronald Labonté

rlabonte@uottawa.ca

1 School of Epidemiology and Public Health, University of Ottawa, 600 Peter Morand Crescent, Ottawa, ON K1G 5Z, Canada

2 Faculty of Medicine, School of Physical and Occupational Therapy, McGill University, Montreal, QC, Canada

3 Faculty of Medicine, University of Zambia, Lusaka, Zambia

4 Economic and Health Policy Research, American Cancer Society, Atlanta, GA, USA

5 Department of Political Science, Marquette University, Milwaukee, WI, USA 